\title{
How to determine the surgical extent in patients with multifocal intraductal papillary mucinous neoplasm?
}

\author{
Sohei SATOI* , Daisuke HASHIMOTO, Tomohisa YAMAMOTO, So YAMAKI, Tatsuma SAKAGUCHI, Mitsugu SEKIMOTO \\ Department of Surgery, Kansai Medical University, Hirakata, Japan
}

Lecture: Intraductal papillary mucinous neoplasms (IPMNs) represent a heterogeneous disease spectrum encompassing benign-to-premalignant-to invasive lesions. IPMNs are cystic precursors to pancreatic ductal adenocarcinoma (PDAC) believed to arise within a widespread neoplastic "field defect". The presence of patients with multifocal disease and/or development of additional lesions over time favorably supports a "field defect theory" and complicates surgical decisions.

The international consensus Fukuoka guideline revealed that all pancreatic ductal epithelial cells are at risk of dysplastic change, as most typically shown in patients with multifocal IPMNs ranging from $18 \%$ to $41 \%$. European guideline proposed that in patients with multifocal IPMNs, each lesion should be evaluated for surgical resection as a single entity. In multifocal IPMNs over the whole pancreatic parenchyma, it may be reasonable to perform partial pancreatectomy to remove the IPMNs with the highest oncological risk and perform life-long surveillance of the remaining lesions. Partial pancreatectomy should achieve margin negative resection with at least low-grade dysplasia margin.

Even if all gross disease can be resected with partial pancreatectomy, there is still the unresolved question of the "field defect" and the extent to which it leaves the pancreatic remnant at risk for recurrent disease. Most pancreatic surgeons agree that the only way to definitively eliminate risk for PDAC is to perform total pancreatectomy (TP). Considering improved glycemic control and pancreatic enzyme replacement therapy, the threshold for TP should perhaps be lowered in patients with high risk as International consensus Fukuoka guideline recommended.

Surgical indication of TP for multifocal IPMNs may be proposed in patients with diffuse main duct involvement, persistent high-grade dysplasia at the resection margin, and familial PDAC. Moreover, pre-operatively diagnosed pancreatobiliary type IPMNs may also serve as a potential target for TP.

Surgical indication of TP should be decided on a case-by-case basis with a risk assessment of the patient considering goals of care and quality of life expectations. 